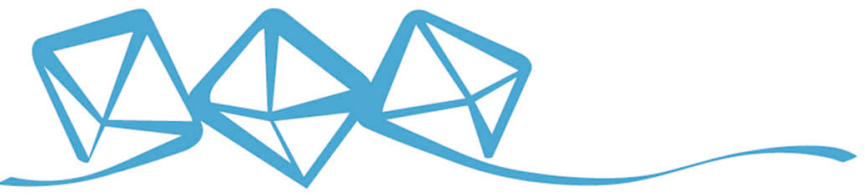 COMMUNICATIONS MATERIALS
}

ARTICLE

https://doi.org/10.1038/s43246-020-00083-1

OPEN

\section{Skyrmion lattice creep at ultra-low current densities}

Yongkang Luo (1) 1,2凶, Shi-Zeng Lin (1) 1, Maxime Leroux', Nicholas Wakeham', David M. Fobes (1) ${ }^{1}$,

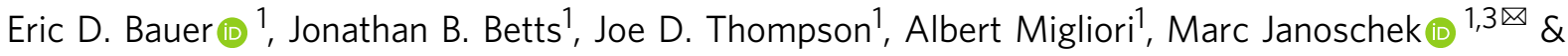
Boris Maiorov (1) ${ }^{1}$

Magnetic skyrmions are well-suited for encoding information because they are nano-sized, topologically stable, and only require ultra-low critical current densities $j_{c}$ to depin from the underlying atomic lattice. Above $j_{c}$ skyrmions exhibit well-controlled motion, making them prime candidates for race-track memories. In thin films thermally-activated creep motion of isolated skyrmions was observed below $j_{c}$ as predicted by theory. Uncontrolled skyrmion motion is detrimental for race-track memories and is not fully understood. Notably, the creep of skyrmion lattices in bulk materials remains to be explored. Here we show using resonant ultrasound spectroscopy - a probe highly sensitive to the coupling between skyrmion and atomic lattices - that in the prototypical skyrmion lattice material MnSi depinning occurs at $j_{c}^{*}$ that is only 4 percent of $j_{c}$. Our experiments are in excellent agreement with Anderson-Kim theory for creep and allow us to reveal a new dynamic regime at ultra-low current densities characterized by thermally-activated skyrmion-lattice-creep with important consequences for applications.

\footnotetext{
${ }^{1}$ Los Alamos National Laboratory, Los Alamos, NM 87545, USA. ${ }^{2}$ Wuhan National High Magnetic Field Center and School of Physics, Huazhong University of Science and Technology, 430074 Wuhan, China. ${ }^{3}$ Laboratory for Neutron and Muon Instrumentation, Paul Scherrer Institute (PSI), 5232

Villigen, Switzerland. ${ }^{凶}$ email: mpzslyk@gmail.com; marc.janoschek@psi.ch
} 
$\mathrm{n}$ materials that exhibit dynamics under the application of external forces, the onset of motion is determined by the underlying pinning landscape. At zero temperature, a welldefined depinning threshold $j_{c}$ exists below which no motion arises, whereas far above $j_{c}$ linear dynamics occur [blue curve in Fig. 1a]. In contrast, at finite temperatures, motion may arise at a much lower threshold $j_{c}^{*}$ [red line in Fig. 1a] due to thermallyactivated creep [Fig. 1b]. Creep is technologically-relevant in systems ranging from structural materials exposed to long-term mechanical stress ${ }^{1}$, to wetting front motion on heterogeneous surfaces $^{2}$, to dynamics of domain walls ${ }^{3}$, and to vortex-motion in superconductors ${ }^{4,5}$.

Early on theory suggested that creep may also be important for magnetic skyrmions ${ }^{6}$. Skyrmions are topologically-stabilized objects with a whirl-like spin-texture that emerge from competing magnetic interactions ${ }^{7}$. Due to their topological stability and solitary particlelike behavior, they pin weakly to defects in the underlying atomic lattice, and substantially lower current densities $j$ are required to move skyrmions compared to magnetic domains. In turn, skyrmions are promising for applications in spintronics and racetrack memory devices based on controlled motion of particle-like magnetic nanostructures ${ }^{8,9}$. Uncontrolled creep motion would be detrimental for devices making the understanding of skyrmion pinning crucial.

Indeed, creep motion of solitary skyrmions in thin films has been already observed ${ }^{10}$. However, in bulk materials, where skyrmions typically form a hexagonal skyrmion lattice (SKX) oriented in a plane perpendicular to an external magnetic field (H) [see Fig. 2a], creep remains elusive. This raises the question whether the pinning mechanisms in thin films and bulk materials are fundamentally different, or if this merely due to differences in pinning landscape. As justified by experiments on both thin films and bulk materials ${ }^{10,12,13}$, skyrmion motion is described by weak collective pinning models ${ }^{6,11}$. For bulk materials, the critical current density $\left(j_{c}\right)$, which denotes the onset of movement is expected to be determined by a trade-off between the strength of the pinning potential $\left[V(x)\right.$, cf Fig. 1b] and the SKX stiffness ${ }^{5}$. Distortion of the SKX in response to pinning centers with a
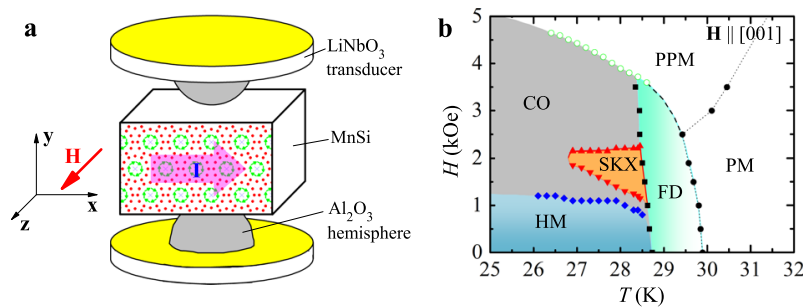

Fig. 2 Resonant ultrasound spectroscopy (RUS) experimental setup and phase diagram of $\mathbf{M n S i}$. a Schematic diagram of the RUS experimental setup. The sample is mounted between two $\mathrm{LiNbO}_{3}$ transducers with the bottom one serving as ultrasonic driving source and the top one as pick-up. The magnetic field $\mathbf{H}$ is applied along [001], and the driving current $\mathbf{I}$ is along [100]. b Magnetic phase diagram established via RUS. The open circles are from $\chi^{\prime}(H)$, defined as the mid-point of spin polarization. The abbreviations denoting the different phases are: $\mathrm{HM}$ helimagnetic, $\mathrm{CO}$ conical, SKX skyrmion lattice, FD fluctuation disordered, PM paramagnetic, and PPM polarized paramagnetic.

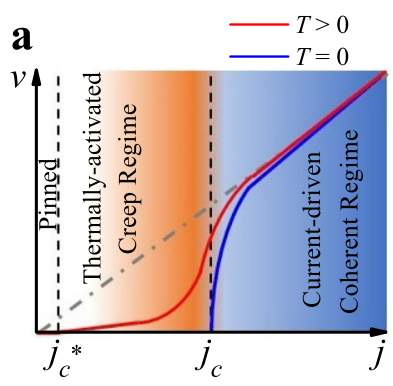

e

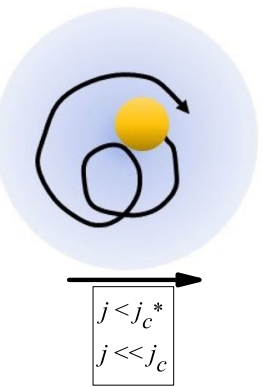

b
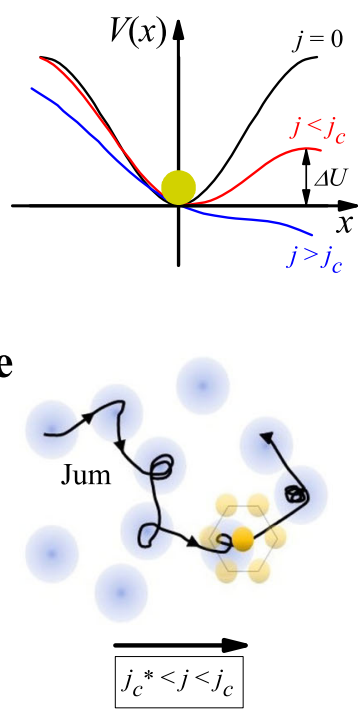

C

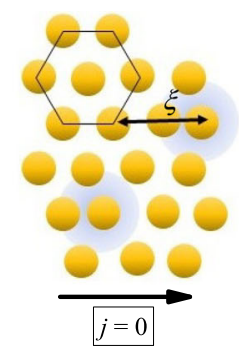

f

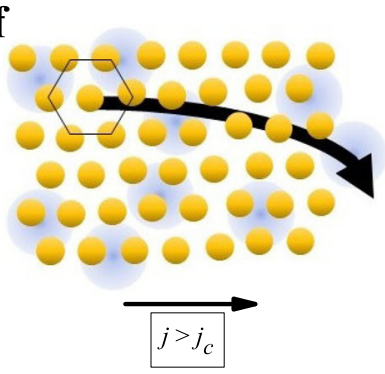

Fig. 1 Skyrmion creep at ultralow current densities. a The dynamic response of skyrmions characterized by a competition of an external drive $j$ (here $j$ is an applied current density) with a pinning potential $V(x)$ [see $\mathbf{b}$ ] can be parametrized via its velocity $v$. This allows to identify three dynamic regimes that are illustrated in d-f and explained in the following. b Local pinning potential $V(x)$ for different current densities. The applied current tilts the pinning potential. Above a critical current density $j_{c^{\prime}}$ the energy barrier $\Delta U$ vanishes completely allowing the skyrmion to depin. At zero temperature $T$ (blue line in a), this well-defined depinning threshold $j_{c}$ thus defines two dynamic regimes, where the skyrmion is either pinned or exhibits current-driven motion. In contrast, for finite $T$ [red curve in $\mathbf{a}$ ], the tilted potential promotes thermal activation of skyrmions already for $j_{c}^{*}<j_{c}$, resulting in a third regime defined by creep. Here $j_{c}^{*}$ is the threshold when skyrmions start to depin from the local pinning center, but remain globally pinned. c A skyrmion lattice (yellow dots) pinned to a few pinning centers (blue shaded areas) is shown. The skyrmion lattice is distorted to accommodate the pinning center, with a characteristic length $\xi$ known as the Larkin length. d For a small current density $j \ll j_{c}$ a thermally activated single skyrmion follows an orbital trajectory around the pinning site because of the Magnus force. e For larger currents $j<j_{c}$ creep may occur. Here the skyrmion spends most of the time orbiting the pinning potential. Further, when the skyrmion escapes from one pinning center due to thermal fluctuations, it will immediately be trapped by a nearby pinning center, resulting in creep motion. f For large current densities $j>j_{c}$, the skyrmion lattice flows freely through the pinning centers and the lattice order is improved as has been previously observed ${ }^{15}$. 
characteristic Larkin length $\xi$ [Fig. 1c] facilitates pinning. Because a perfectly rigid SKX cannot be pinned, $j_{c}$ depends inversely on the stiffness.

The application of a driving current tilts the pinning potential. At zero temperature, skyrmions escape only when $\Delta U \leq 0$ [Fig. 1b], where $\Delta U$ is the height of the tilted $V(x)$. In contrast, for finite temperature, the escape rate from pinning sites due to thermal fluctuations is given by $\Gamma \propto \exp \left(-\Delta U / k_{\mathrm{B}} T\right)$, where $k_{\mathrm{B}}$ is Boltzmann constant. When the external drive is small, a thermally-activated skyrmion follows an orbital trajectory around the pinning site due to the Magnus force [see Fig. 1d]. For $j_{\mathrm{c}}^{*}<j_{\mathrm{c}}$, a skyrmion may escape from a pinning center due to thermal fluctuations; however, it will immediately be trapped by a nearby pinning center ${ }^{6}$, resulting in creep [Fig. le]. Here $j_{c}^{*}$ is a threshold current that denotes that the energy barrier $\Delta U$ of the local pinning potential is smaller than the energy $k_{\mathrm{B}} T$ of thermal fluctuations. Effectively, skyrmions hop between pinning centers aided by thermal fluctuations, resulting in nonlinear motion ${ }^{11}$. The exact creep path depends on the type and distribution of such pinning centers. For SKX thermal fluctuations may depin small fractions of the lattice [shown in light yellow in Fig. 1e], where each fraction encounters a distinct local pinning landscape, in turn, resulting in incoherent movement characteristic of local creep. With increasing $j$, and thus decreasing $\Delta U$, the fraction of the SKX that may be depinned by fluctuation becomes larger. Eventually global creep occurs, for which the entire SKX becomes depinned and repinned continuously due to a small but nonzero $\Delta U$ that allows to recapture the SKX. For $j \gg j_{\mathcal{c}}$, which denotes that $\Delta U<0$, the SKX flows freely through pinning centers [Fig. 1f], leading to a linear regime with coherent motion of the entire SKX [Fig. 1a].

The observation of creep motion of solitary skyrmions in thin films was achieved via polar magneto-optical Kerr effect (MOKE) microscopy using current pulses ${ }^{10}$. MOKE also was used to probe skyrmion diffusion, which is interesting in its own right as it has potential for applications in probabilistic computing, was recently observed in a thin film heterostructure that was engineered to have low $\Delta U^{14}$. Thermal diffusion of skyrmions can arise at zero current at elevated temperatures that entail a thermal energy $k_{\mathrm{B}} T$ larger than the unaltered energy barrier $\Delta U(j=0)$. However, the spatial resolution of MOKE is not sufficient to probe SKX motion, because skyrmions in bulk materials are substantially smaller $(10-100 \mathrm{~nm})$ than in thin films $(\mu \mathrm{m})$.

Instead, in bulk materials, microscopic skyrmion movement under current has been investigated by Lorentz transmission electron microscopy (LTEM) $)^{15}$ and via spin-transfer torque over the lattice by small-angle neutron scattering (SANS) ${ }^{12}$. Macroscopically, it has also been inferred from a reduction of the topological Hall effect (THE) ${ }^{16,17}$. However, apart from the associated macroscopic $j_{\mathfrak{c}}$, no detailed information on the depinning process is available. In particular, the Hall effect is not suited to identify creep because it provides skyrmions with sufficient time to relax into equilibrium positions on the pinning sites, and thus eliminates side-jump motion caused by the Magnus force $e^{11}$.

To reveal previously elusive SKX creep in bulk materials we exploit the extreme sensitivity of resonant ultrasound spectroscopy (RUS) to the coupling between the SKX and the atomic lattice ${ }^{18}$. RUS probes the resonant frequencies $F_{\mathrm{i}}$ of a solid which depend on its elasticity to determine the complete elastic tensor (C). Details of how $\mathbf{C}$ is computed from the measured $F_{\mathrm{i}}$ are described in supplementary note 1 (cf. Fig. S1 and Table S1). RUS experiments under applied current on the prototypical SKX material $\mathrm{MnSi}$ allow us to probe the depinning of SKX with unprecedented resolution, and thus to determine $j_{\mathrm{c}}^{*}$ directly [see Fig. 2a and "Methods" section]. We find that in MnSi creep motion occurs at a critical current density $j_{c}^{*}$ that is only $4 \%$ of $j_{c}$. We show that our experimental results are in excellent agreement with Anderson-Kim theory for $\mathrm{creep}^{19}$, and connect the creep motion of skyrmion lattices in bulk materials with the previously known creep dynamics in thin films.

\section{Results}

Elastic response to the formation of the Skyrmion lattice. First, we briefly discuss RUS experiments at $j=0$, which establish the magnetic phase diagram [Fig. 2b], consistent with AC magnetic susceptibility $\left(\chi^{\prime}\right)$ (see Supplementary Note 2, Fig. S2) and literature ${ }^{7}$. The SKX phase appears in a narrow range of temperatures and field within the conically $(\mathrm{CO})$ ordered magnetic phase. A fluctuation-disordered (FD) region ${ }^{20}$ can also be seen right above $T_{\mathrm{c}}=28.7 \mathrm{~K}$ where the system undergoes a paramagnetic-helimagnetic (PM-HM) transition. In the absence of magnetic field, due to the cubic symmetry of $\mathrm{MnSi}$, only three independent elastic moduli $C_{11}, C_{12}$, and $C_{44}$ are required. This is corroborated by our measurements (see Fig. S3a-c in Supplementary Note 3). Under magnetic field $\mathbf{H} \|[001]$, the symmetry of the elastic tensor is lowered to tetragonal (Supplementary Note 4), requiring three additional independent elastic moduli, $C_{33}, C_{23}\left(=C_{31}\right)$ and $C_{66}$. In Fig. 3a-c, we plot $C_{i j}$ as a function of $H$ at $T=28 \mathrm{~K}$. Each subset of $C_{i j}$ splits into two branches under magnetic field. We observe a discontinuous jump in some $C_{i j}$ between $H_{a 1}=1.4 \mathrm{kOe}$ and $H_{a 2}=2.2 \mathrm{kOe}$. Based on the field dependence of $\chi^{\prime}$ shown in Fig. S2b (Supplementary Note 2), we determine $H_{a 1}$ and $H_{a 2}$ as the lower and upper boundaries of the SKX phase, respectively. Below $H_{a 1}$, there is a weak inflection in $C_{i j}(H)$ near $H_{c 1}=1.0 \mathrm{kOe}$, assigned as the field-induced HM-CO phase transition. Note that the elastic response to the SKX phase has not been seen previously ${ }^{21,22}$ in off-diagonal moduli $C_{i j}$ $(i \neq j)$. Because RUS probes all $C_{i j}$ in a single frequency sweep ${ }^{23,24}$ it directly reveals the shear modulus $C^{*} \equiv\left(C_{11}-C_{12}\right) / 2$, which exhibits a much smaller variation than the compression moduli. Further, the jump in $C^{*}$ is an order of magnitude bigger than in $C_{66}$, indicating that hexagonal symmetry of the SKX does not describe the system, as this requires $C^{*}=C_{66}$ (see Supplementary Note 4). Instead, this shows that RUS probes the response of the chemical lattice (which is tetragonal in field) to the formation of the hexagonal SKX.

We note that when tracking changes and discontinuities, it is more reliable to plot the raw frequencies ${ }^{25}$. As shown in Table S1 in Supplementary Note 1, the two resonances $F_{1654}$ and $F_{2419}$ are predominantly related to $C_{11}$. For a frequency that depends only on one $C_{i j}$ we have $C_{i j} \propto F^{2}$, so for small changes in $C_{i j}$ we have $\delta C_{i j} \sim 2 \delta F$. Figure 3e displays the temperature dependence of $F_{2419}$ at various magnetic fields. For $H=0$, the profile of $F_{2419}(T)$ resembles that of $C_{11}(T)$ [Fig. S4a], confirming the dominance of $C_{11}$. With increasing magnetic field, $T_{\mathrm{c}}$ is gradually suppressed, and the signature of the phase transition becomes more pronounced for $H>2.5 \mathrm{kOe} T^{*}$, the temperature where the minimum of $F_{2419}(T)$ occurs, initially decreases with increasing $H$ but then broadens and shifts to higher $T$ for $H>2.5 \mathrm{kOe}$ where spins become polarized by the external field. The window between $T^{*}$ and $T_{\mathrm{c}}$ describes the FD region in Fig. $2 \mathrm{~b}$. Figure $3 \mathrm{f}$ shows $F_{1654}$ as a function of $H$ measured at selected temperatures. The discontinuous jump in $F_{1654}(H)$ can be identified between 26.9 and $28.5 \mathrm{~K}$ similarly as seen in $C_{11}$ in Fig. 3a. A positive jump in $F_{1654}(H)$ signifies stiffening in $C_{11}$ when the system enters the SKX phase. The (maximal) magnitude of the jump in $F_{1654}(H)$, denoted by $\Delta F$ [see Fig. 4e], is plotted as a function of temperature in Fig. $4 \mathrm{~g}$ with a maximum near the SKX-FD boundary and decreasing as $T$ decreases. The value of $\Delta F$, therefore, is a qualitative measure for the coupling between 

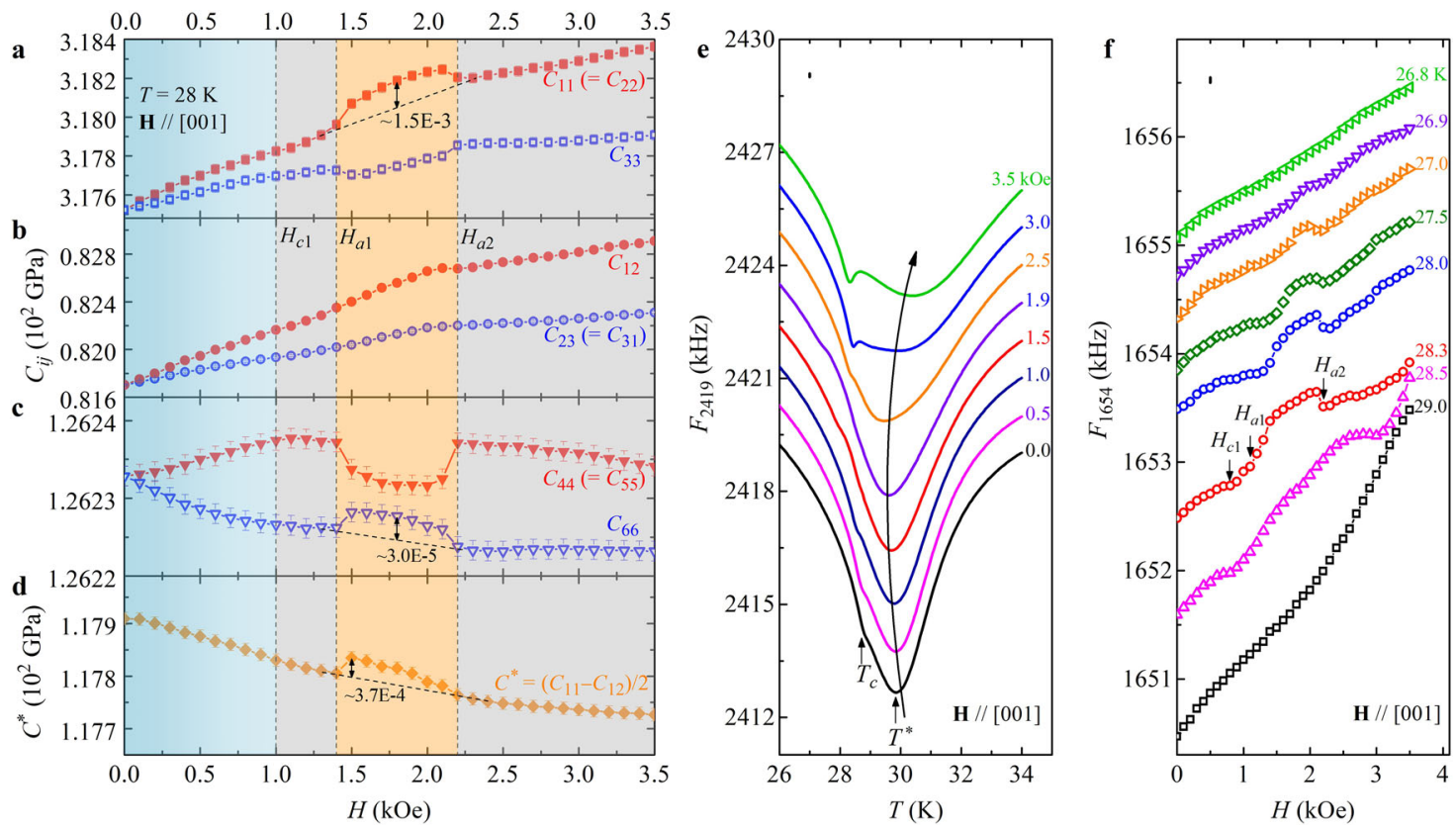

Fig. 3 Elastic properties of $\mathbf{M n S i}$ at $\boldsymbol{j}=\mathbf{0}$ determined by resonant ultrasound spectroscopy (RUS). a-d Isothermal elements $C_{i j}$ and $C^{\star} \equiv\left(C_{11}-C_{12}\right) / 2$ of the elastic tensor $\mathbf{C}$ as a function of magnetic field $H$, respectively. Although both compression $\left(C_{11}, C_{33}\right)$ and shear moduli $\left(C_{44}\right.$, $\left.C_{66}\right)$ display abrupt changes, $C_{12}$ and $C_{23}$ only exhibit a slight change in slope near $H_{a 1}$ and $H_{a 2}$. The accuracy of the absolute values of the $C_{i}$ is determined by the quality of the fits of the resonance frequencies, which is of the order of $0.1 \%$ (see ref. 24 and table S1 in Supplementary note 1)). However, our results rely on relative shifts of the $C_{i}$, which can be determined with an accuracy of better than $0.01 \%$, as indicated by the error bars. e Temperature ( $T$ ) dependence of the resonant frequency $F_{2419}$ measured for various $H . T_{\mathrm{c}}$ is the critical temperature for the paramagnetic (PM) to helimagnetic (HM) transition, and $T^{*}$ is the characteristic temperature below which the fluctuation-disordered (FD) regime arises. $\mathbf{f} F_{1654}$ as a function of $H$, measured at selected temperatures $T$. The accuracy of determining relative frequency shifts with our RUS setup is better than $0.01 \%$ as denoted by the black bar in the upper left corner of the panels $\mathbf{e}, \mathbf{f}^{24}$
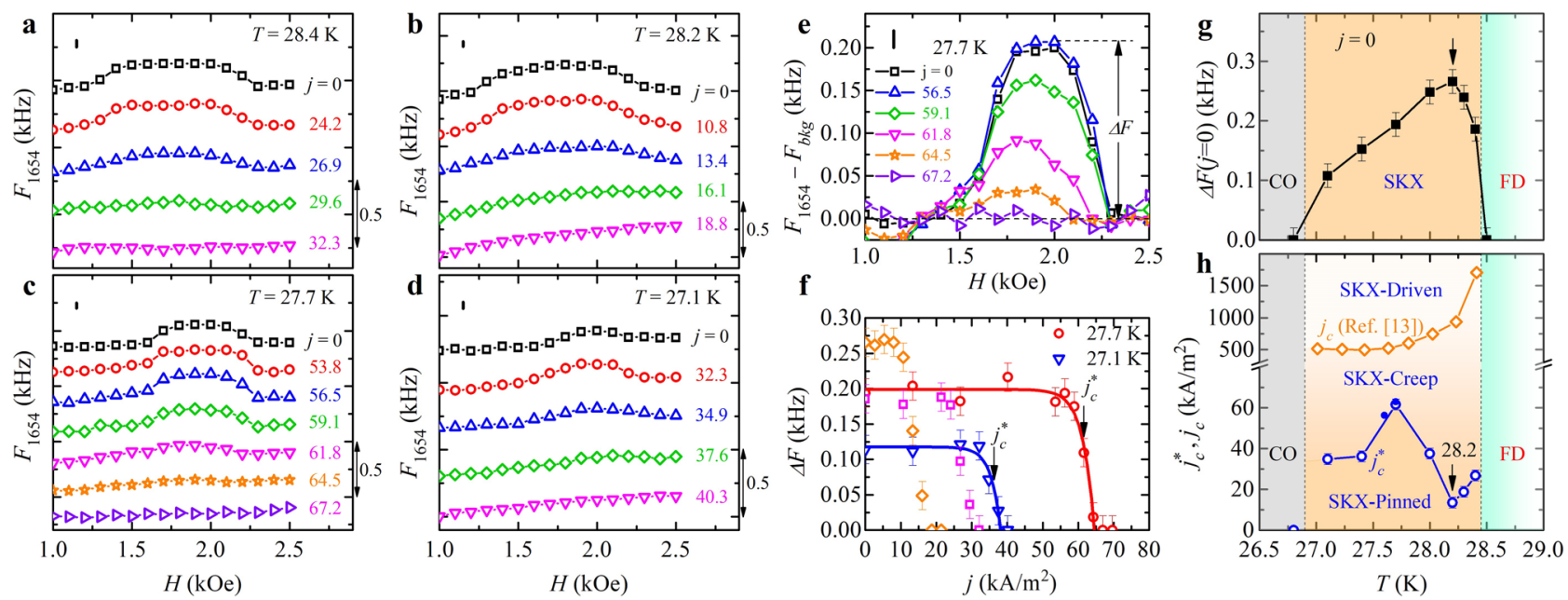

Fig. 4 Signatures of skyrmion creep in the elastic properties. a-d Field dependence of the resonance frequency $F_{1654}$ at various current densities $j$, measured at temperatures $T=28.4 \mathrm{~K}, 28.2 \mathrm{~K}, 27.7 \mathrm{~K}$ and $27.1 \mathrm{~K}$, respectively. The curves are vertically offset for clarity. The double arrow on the right side of each panel denotes the scale of the frequency shift. The accuracy of determining relative frequency shifts with our RUS setup is better than $0.01 \% 24$ as denoted by the black bar in the upper left corner of the panels a-d. e $F_{1654}-F_{\text {bkg }}$ as a function of $H$ at $27.7 \mathrm{~K}$, where $F_{\text {bkg }}$ is the smooth background of $F_{1654} . \Delta F$ is defined as the maximum of $F_{1654}-F_{\text {bkg. }} \mathbf{f} \Delta F$ vs. $j$ for $27.1,27.7,28.2$, and $28.4 \mathrm{~K}$. The solid lines are fits to Anderson-Kim model, $\Delta F(j)-\Delta F(j=0) \propto \exp \left[\beta\left(j-j_{c}^{*}\right) / k_{B} T\right]$, where $\beta$ is material dependent a prefactor. In the Anderson-Kim model the local pinning potential vanishes linearly with current $\Delta U=\beta\left(j-j_{c}^{*}\right)$. The arrows marks the critical current density value $j_{c}^{*}=62 \mathrm{kA} / \mathrm{m}^{2}$ for $27.7 \mathrm{~K}$ to illustrate how critical current densities where determined. $\mathbf{g} \Delta F$ as a function of $T$ in the absence of current. $\mathbf{h}$ Temperature dependent $j_{c}^{*}$ (blue line and symbols). The full symbols are measured with an ultrasonic excitation two times larger than that for the open symbols. The orange line and symbols are reproduced from ref. ${ }^{13}$ and denote the onset of coherent linear skyrmion lattice motion as determined by the reeduction of the topological Hall effect (THE) under current. 
chemical lattice and SKX. Indeed, the maximum in $\Delta F$ near the upper boundary of the SKX $(T \sim 28.2 \mathrm{~K})$ phase can be explained by proximity to the FD regime (see below).

Elastic response under applied current. Now that we have established the elastic response to the presence of the SKX, we demonstrate the influence of applied electrical current. Figure 4a-d display the field dependence of $F_{1654}$ for various applied electrical currents $(\mathbf{I} \perp \mathbf{H})$ at four selected temperatures 28.4, 28.2, 27.7, and $27.1 \mathrm{~K}$ inside the SKX phase, respectively. We note that the resonance $F_{1654}$ is most sensitive to the presence of the SKX because it is related to the elastic moduli $C_{11}$, which is more than an order of magnitude larger than any other $C_{i j}$. In addition, $F_{1654}$ provides the highest quality signal as described in more detail in Supplementary Note 1 . Taking $T=27.7 \mathrm{~K}$ as an example [Fig. 4c], $\Delta F$ remains essentially unchanged for $j$ up to $56.5 \mathrm{kA} / \mathrm{m}^{2}$, drops abruptly between 59.1 and $64.5 \mathrm{kA} / \mathrm{m}^{2}$, and becomes nearly unresolvable at $67.2 \mathrm{kA} / \mathrm{m}^{2}$, as if the SKX is completely decoupled from the lattice. The threshold current density $j_{c}^{*}$ is defined as the midpoint of the drop in $\Delta F$, shown in Fig. $4 \mathrm{f}$ with $j_{\mathrm{c}}^{*}=62(3) \mathrm{kA} / \mathrm{m}^{2}$ at $27.7 \mathrm{~K}$. The error bar is set by the step size in current. In Fig. $4 \mathrm{f}$ we also show $\Delta F(j)$ for $27.1 \mathrm{~K}$, and the difference in $j_{\mathrm{c}}^{*}$ is far larger than the measurement uncertainty. We emphasize that the changes observed in the resonance frequencies are not caused by current-induced Joule heating as can be illustrated by several observations. (i) The magnitude of $\Delta F$ does not increase with $j$ [cf Fig. 4f] for temperatures near the lower boundary of the SKX phase (e.g., $27.1 \mathrm{~K}$ ); (ii) $j_{\mathrm{c}}^{*}$ does not increase monotonically with decreasing temperature; (iii) the phase boundaries in $H$ depend strongly on temperature as revealed in Fig. 2b, but the width of the SKX phase with respect to field showing nonzero $\Delta F$ remains unaffected for increasing $j$ at all measured temperatures, as expected for constant temperature [Fig. 4a-e].

\section{Discussion}

The drastic changes in the elastic properties above $j_{\mathrm{c}}^{*}$ suggest that skyrmion depinning occurs at critical current densities that are a factor of 25 smaller than $j_{\mathrm{c}} \sim 1.5 \mathrm{MA} / \mathrm{m}^{2}$ derived from previous measurements $12,13,15,17,26$. There are several scenarios that may explain this disparity. First, the presence of ultrasonic waves could facilitate depinning by shaking skyrmions off pinning potentials yielding a smaller current for motion threshold as previously observed for superconducting vortices ${ }^{27}$. However, this effect is unlikely here because the same $j_{\mathrm{c}}^{*}$ is observed with ultrasonic excitation with twice the amplitude [full symbols in Fig. 4h]. Another possibility is the difference in pinning defects in our sample compared to previous studies. However, a detailed characterization of our sample (see Supplementary Note 5 and Fig. S4) reveals that it is of the same high-quality as samples used in previous studies $7,12,13,20$, as notably demonstrated by a large residual resistivity ratio $(R R R=87)$.

As we discuss in the following, a consistent view on the difference between $j_{\mathrm{c}}$ and $j_{\mathrm{c}}^{*}$ may be established by considering the different sensitivity in detecting the onset of skyrmion motion with distinct techniques. As explained in the introduction, THE is unable to measure incoherent skyrmion motion resulting from creep. Similarly, SANS is only sensitive to coherent rotation of the entire SKX due to spin-transfer torque ${ }^{12}$. In contrast, RUS directly measures the magneto-crystalline coupling, and thus detects skyrmion movement immediately when the SKX decouples from the atomic lattice. Thus, it can detect motion due to creep at much lower current density [cf. Fig. 1a] as corroborated by the abrupt change in $\Delta F$ as $j$ reaches $j_{c}^{*}$ that is contrasted by the gradual decrease of the topological Hall resistivity for $j>j_{c}{ }^{13}$. This is similar to superconducting vortices, where magnetization measurements and electrical transport are sensitive to creep and flux-flow changes, respectively $y^{5,28,29}$.

That $j_{\mathrm{c}}^{*}$ marks the presence of a creep regime is also evidenced by the fits of our data to Anderson-Kim theory for creep for which the local pinning potential vanishes linearly with current $\Delta U=\beta\left(j-j_{\mathrm{c}}^{*}\right)$, where $\beta$ is a prefactor ${ }^{19}$. Notably, the measured $\Delta F$ is well-described by $\Delta F(j)-\Delta F(j=0) \propto \exp \left[\left(j-j_{\mathrm{c}}^{*}\right) \beta / k_{\mathrm{B}} T\right]$ over the entire temperature range of the SKX phase [Fig. $4 \mathrm{f}]$. This creep scenario is further in agreement with the observed temperature dependence of $j_{c}^{*}$. As described above, according to weak collective pinning theory, $j_{\mathrm{c}}^{*}$ is inversely proportional to the SKX stiffness. The temperature dependence of $\Delta F(j=0)$ [see Fig. 4g] displays two trends. Starting at high temperature from the SKXFD boundary, $\Delta F$ initially increases as $T$ decreases becoming stiffer down to $T=28.2 \mathrm{~K}$, where $j_{\mathrm{c}}^{*}$ also minimizes [vertical arrows in Fig. 4g, h]. The behavior for $T>28.2 \mathrm{~K}$ is consistent with strong thermal fluctuations near the upper boundary of the SKX phase that soften the SKX lattice which allows to better accommodate local pinning sites, in turn, improving pinning. The resulting enhancement of $j_{c}^{*}$ near the SKX-FD phase boundary is called peak effect and was also observed in $\mathrm{MnSi}$ via the $\mathrm{THE}^{13}$ [see orange diamond symbols and line in Fig. 4h] and is welldocumented for superconducting vortices ${ }^{5,30}$. As $T$ continues to decrease, $j_{c}^{*}$ and $\Delta F(j=0)$ keep displaying an inverse relation consistent with weak collective pinning down to $T=27.7 \mathrm{~K}$, where $j_{\mathrm{c}}^{*}(T)$ shows a maximum but $\Delta F(T)$ is featureless.

To understand the behavior below $T=27.7 \mathrm{~K}$, it is important to consider that for bulk materials such as $\mathrm{MnSi}$, the size and shape of the SKX phase is sensitive to the sample geometry due to demagnetization effects ${ }^{31}$. In addition, it has been shown via neutron diffractive imaging that the phase transition to the conical phase is characterized by macroscopic phase separation where only parts of the sample show SKX order, whereas the rest exhibits conical order ${ }^{32}$. In addition, where in the sample the SKX phase nucleates (edge vs. center) strongly varies as a function of magnetic field $^{32}$. For plate-like samples as were required for our combined RUS and current study, the influence of demagnetization fields is particularly strong in the part of SKX phase that is characterized by $T<27.7 \mathrm{~K}$ as the macroscopic phase separation is observed for more than $50 \%$ of the field range of the SKX phase $\mathrm{s}^{31,32}$. The fraction of the SKX phase showing phase separation increases further when the temperature is lowered ${ }^{31,32}$. Naturally, the prominent phase separation in the low-temperature regime of the SKX phase, results in magnetic domain boundaries between the conical and SKX phases, which influences the pinning of the SKX. This distinct pinning regime is reflected in a change of the behavior of $j_{\mathrm{c}}^{*}(T)$ for $T<27.7 \mathrm{~K}$.

It is interesting to compare the SKX creep in the bulk material $\mathrm{MnSi}$ identified here to the creep of individual skyrmions observed in thin films reported previously ${ }^{10}$. The ratio of $j_{\mathrm{c}} / j_{\mathrm{c}}^{*}$ found for $\mathrm{MnSi}$ varies between 10 and 50 depending on temperature, which is a factor 10-20 larger than the ratio in thin films. Because both the onset of creep at $j_{c}^{*}$ and the onset of coherent motion at $j_{c}$ depend on the pinning potential, it is unlikely that this difference is due to a difference in amount and nature of the defects that pin skyrmions in bulk and thin film materials, respectively. Instead this difference originates from a skyrmion lattice being easier to recapture by pinning sites compared to single skyrmions, and therefore a substantially larger $j_{c} / j_{c}^{*}$ is needed to enter the linear driven regime. Finally, in contrast to single skyrmions, for SKX we can also differentiate local and global creep motion. As discussed above $j_{c}^{*}$ denotes the onset of local creep where only part of the SKX is depinned. Our RUS measurements demonstrate that for current densities $j$ that are a only few percent larger than $j_{\mathrm{c}}^{*}, \Delta F$ vanishes. Because $\Delta F$ is a 
measure of coupling between the SKX and the underlying lattice, this suggest a crossover from local to global creep, where on average the SKX is depinned, but is recaptured continuously.

In conclusion, our RUS measurements on the prototypical SKX material $\mathrm{MnSi}$ under applied electrical currents, provide evidence for the existence of a novel regime of skyrmion lattice dynamics in bulk materials at substantially lower depinning current densities $j_{\mathrm{c}}^{*}$ than previously reported for thin films. The temperature dependence of this new intermediate regime is consistent with thermally induced creep of a skyrmion lattice. Our results directly connect the creep motion of skyrmion lattices in bulk materials with the known creep dynamics in thin films, showing that the underlying assumptions for a tilted local weak pinning potential ${ }^{6,11}$ is the correct model for both cases despite obvious differences in the interactions that support the emergence of skyrmions. This highlights that our current theoretical understanding of skyrmion creep is complete, and will be relevant for applications. Notably, because $j_{\mathrm{c}}^{*}$ is only about four percent of $j_{\mathrm{c}}$ above which coherent skyrmion motion occurs, it is crucial that any devices based on the control of skyrmion dynamics must be carefully engineered to avoid uncontrolled creep. This is particularly critical as real devices will have to work at room temperature, where thermally activated creep will be substantial.

\section{Methods}

The MnSi single crystal was grown by the Bridgman-Stockbarger method followed by a 1-week anneal at $900^{\circ} \mathrm{C}$ in vacuum. The stoichiometry of the crystal was examined by energy dispersive $\mathrm{x}$-ray spectroscopy (EDS). The sample was polished into a parallelepiped along the [001] direction with dimensions $1.446 \times 0.485 \times 0.767 \mathrm{~mm}^{3}$. The orientation of the crystal was verified by Laue X-ray diffraction within $1^{\circ}$. Electrical resistivity and AC susceptibility measurements revealed a magnetic transition as expected at $T_{\mathrm{c}}=28.7 \mathrm{~K}$, and a residual resistance ratio $R R R[\equiv \rho(300 \mathrm{~K}) / \rho$ $(T \rightarrow 0)]=87$, indicating a high quality single crystal (see Fig. S2 in Supplemenatry Note 2). All the measurements in this work were performed on the same crystal. Further, the SKX in a different piece of this sample was directly observed using SANS $^{33}$.

A schematic diagram of the RUS setup is shown in Fig. 2a. The sample was mounted between two $\mathrm{LiNbO}_{3}$ transducers. In order to stabilize the sample in a magnetic field and maintain RUS-required weak transducer contact, $\mathrm{Al}_{2} \mathrm{O}_{3}$ hemispheres (that also act as wear plates and electrical insulators) were bonded to each transducer. The external magnetic field $\mathbf{H}$ was applied along [001] of the cubic crystal structure of MnSi. Frequency sweeps from 1250 to $5300 \mathrm{kHz}$ were performed for each measurement. The resonance peaks were tracked and recorded as a function of temperature and magnetic field. Elastic moduli $C_{i j}$ were extracted from 24 resonance frequencies with an RMS error of $0.2 \%$ using an inversion algorithm ${ }^{23,24}$. Although the absolute error is large (mainly because of uncertainties in the sample dimensions), the precision of the elastic moduli determination is at least $1 \times 10^{-7}$. Finally, we note that by measuring all the elastic moduli $C_{i j}$ simultaneously with a fixed magnetic field orientation, accounting for anisotropic demagnetization factors is unnecessary and direct comparisons among $C_{i j}$ can be made.

To study the effect of current on moduli, we attached gold wires $(13 \mu \mathrm{m})$ at opposite sides of the specimen allowing a DC current to be applied along [100], I $\perp \mathbf{H}$ with a cross-section of $0.485 \times 0.767 \mathrm{~mm}^{2}$. To minimize Joule heating at the contacts, the Au wires were spot-welded to the sample and covered with silver paint to improve current homogeneity and reduce contact resistance. The resulting Ohmic electrical contacts were less than $0.5 \Omega$. Whenever the current was changed, we waited for steady state before recording. A small temperature increase $(<30 \mathrm{mK})$ was observed at the thermometer right after applying relatively larger currents. We compensated for this by adjusting the set-point of the temperature controller.

\section{Data availability}

The data that support the plots within this paper and other findings of this study are available from the corresponding author upon reasonable request.

\section{Code availability}

The algorithm used to fit the RUS data is detailed in the Supplementary Note 2. The computer code itself is available from the corresponding author upon reasonable request.

Received: 20 January 2020; Accepted: 12 October 2020;

Published online: 11 November 2020

\section{References}

1. Courtney, T. H. Mechanical Behavior of Materials. (Waveland Press, Illinois, 2005).

2. Alava, M., Dubé, M. \& Rost, M. Imbibition in disordered media. Adv. Phys. $\mathbf{5 3}$ 83-175 (2004).

3. Cayssol, F., Ravelosona, D., Chappert, C., Ferré, J. \& Jamet, J. P. Domain wall creep in magnetic wires. Phys. Rev. Lett. 92, 107202 (2004).

4. Larkin, A. I. \& Ovchinnikov, Y. N. Pinning in type II superconductors. J. Low Temp. Phys. 34, 409-428 (1979).

5. Blatter, G., Feigeloman, M. V., Geshkenbein, V. B., Larkin, A. I. \& Vinokur, V. M. Vortices in high-temperature superconductors. Rev. Mod. Phys. 66, 1125-1388 (1994)

6. Lin, S. Z., Reichhardt, C., Batista, C. D. \& Saxena, A. Particle model for skyrmions in metallic chiral magnets: Dynamics, pinning, and creep. Phys. Rev. B 87, 214419 (2013).

7. Mühlbauer, S. et al. Skyrmion lattice in a chiral magnet. Science 323, 915-919 (2009).

8. Fert, A., Cros, V. \& Sampaio, J. Skyrmions on the track. Nat. Nanotech 8, 152-156 (2013).

9. Müller, J. et al. Magnetic skyrmions on a two-lane racetrack. New J. Phys. 19, 025002 (2017)

10. Jiang, W. et al. Direct observation of the skyrmion Hall effect. Nat. Phys. 13, 162-169 (2017).

11. Reichhardt, C. \& Reichhardt, C. J. O. Thermal creep and the skyrmion Hall angle in driven skyrmion crystals. Matter 31, 07LT01 (2019).

12. Jonietz, F. et al. Spin transfer torques in $\mathrm{MnSi}$ at ultralow current densities. Science 330, 1648-1651 (2010).

13. Schulz, T. et al. Emergent electrodynamics of skyrmions in a chiral magnet. Nat. Phys. 8, 301-304 (2012).

14. Zázvorka, J. et al. Thermal skyrmion diffusion used in a reshuffler device. Nat. Nanotechnol. 14, 658-661 (2019).

15. Yu, X. Z. et al. Skyrmion flow near room temperature in an ultralow current density. Nat. Commun. 3, 988 (2012).

16. Reichhardt, C., Ray, D. \& Reichhardt, C. J. O. Collective transport properties of driven skyrmions with random disorder. Phys. Rev. Lett. 114, 217202 (2015).

17. Dong, L. et al. Current-driven dynamics of skyrmions stabilized in $\mathrm{MnSi}$ nanowires revealed by topological Hall effect. Nat. Commun. 6, 8217 (2015).

18. Luo, Y. et al. Anisotropic magnetocrystalline coupling of the skyrmion lattice in MnSi. Phys. Rev. B 97, 104423 (2018).

19. Anderson, P. W. \& Kim, Y. B. Hard superconductivity: theory of the motion of abrikosov flux lines. Rev. Mod. Phys. 36, 39-43 (1964).

20. Janoschek, M. et al. Fluctuation induced first-order phase transition in Dzyaloshinskii-Moriya helimagnets. Phys. Rev. B 87, 134407 (2013).

21. Nii, Y., Kikkawa, A., Taguchi, Y., Tokura, Y. \& Iwasa, Y. Elastic stiffness of a skyrmion crystal. Phys. Rev. Lett. 113, 267203 (2014).

22. Petrova, A. E. \& Stishov, S. M. Field evolution of the magnetic phase transition in the helical magnet MnSi inferred from ultrasound studies. Phys. Rev. B 91, 214402 (2015).

23. Migliori, A. \& Sarrao, J. L. Resonant Ultrasound Spectroscopy: Applications to Physics, Materials Measurements, and Nondestructive Evaluation. (Wiley, New York, 1997).

24. Migliori, A. et al. Resonant ultrasound spectroscopic techniques for measurement of the elastic moduli of solids. Phys. B 183, 1-24 (1993).

25. Evans, D. et al. Defect dynamics and strain coupling to magnetization in the cubic helimagnet $\mathrm{Cu}_{2} \mathrm{OSeO}_{3}$. Phys. Rev. B 95, 094426 (2017).

26. Leroux, M. et al. Skyrmion lattice topological hall effect near room temperature. Sci. Rep. 8, 15510 (2018).

27. Valenzuela, S. O. \& Bekeris, V. Oscillatory dynamics and organization of the vortex solid in $\mathrm{YBa}_{2} \mathrm{Cu}_{3} \mathrm{O}_{7}$ single crystals. Phys. Rev. Lett. 86, 504-507 (2001).

28. Maiorov, B. et al. Synergetic combination of different types of defect to optimize pinning landscape using $\mathrm{BaZrO}_{3}$-doped $\mathrm{YBa}_{2} \mathrm{Cu}_{3} \mathrm{O}_{7}$. Nat. Mater. 8, 398-404 (2009).

29. Campbell, A. M. \& Evetts, J. E. Critical currents in superconductors. $A d v$. Phys. 50, 1249-1449 (2001).

30. Valenzuela, S. O., Maiorov, B., Osquiguil, E. \& Bekeris, V. Elastic-to-plastic crossover below the peak effect in the vortex solid of $\mathrm{YBa}_{2} \mathrm{Cu}_{3} \mathrm{O}_{7}$ single crystals. Phys. Rev. B 65, 060504 (2002).

31. Bauer, A. \& Pfleiderer, C. Magnetic phase diagram of MnSi inferred from magnetization and ac susceptibility. Phys. Rev. B 85, 214418 (2012).

32. Reimann, T. et al. Neutron diffractive imaging of the skyrmion lattice nucleation in MnSi. Phys. Rev. B 97, 020406 (2018).

33. Fobes, D. et al. Versatile strain-tuning of modulated long-period magnetic structures. Appl. Phys. Lett. 110, 192409 (2017).

\section{Acknowledgements}

We thank F. F. Balakirev for technical support, and F. Ronning and M. Garst for insightful conversations. Sample synthesis by EDB and characterization by JDT was performed under 
the U.S. DOE, Office of Science, BES project "Quantum Fluctuations in Narrow Band Systems”. Research by Y.L., S.L., D.M.F., M.L., N.D., B.M., and M.J. was supported by LANL Directed Research and Development program project "A New Approach to Mesoscale Functionality: Emergent Tunable Superlattices (20150082DR)” [PI Janoschek]. Work by J.B. and A.M. was part of the Materials Science of Actinides, an Energy Frontier Research Center funded by the U.S. DOE, Office of Science, BES under Award DE-SC0001089. Y.L. acknowledges support by the 1000 Youth Talents Plan of China.

\section{Author contributions}

Y.L., S.L., M.J., and B.M. conceived and designed the experiments. EDB synthesized the samples. M.L., N.W., D.M.F., and J.D.T. characterized the crystals. J.B. and A.M. provided technical support for the experimental set-up. Y.L. performed most of the ultrasonic measurements. Y.L., S.L., M.J., and B.M. discussed the data, interpreted the results, and wrote the paper with input from all the authors.

\section{Competing interests}

The authors declare no competing interests.

\section{Additional information}

Supplementary information is available for this paper at https://doi.org/10.1038/s43246020-00083-1.
Correspondence and requests for materials should be addressed to Y.L. or M.J.

Reprints and permission information is available at http://www.nature.com/reprints

Publisher's note Springer Nature remains neutral with regard to jurisdictional claims in published maps and institutional affiliations.

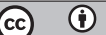

Open Access This article is licensed under a Creative Commons Attribution 4.0 International License, which permits use, sharing, adaptation, distribution and reproduction in any medium or format, as long as you give appropriate credit to the original author(s) and the source, provide a link to the Creative Commons license, and indicate if changes were made. The images or other third party material in this article are included in the article's Creative Commons license, unless indicated otherwise in a credit line to the material. If material is not included in the article's Creative Commons license and your intended use is not permitted by statutory regulation or exceeds the permitted use, you will need to obtain permission directly from the copyright holder. To view a copy of this license, visit http://creativecommons.org/ licenses/by/4.0/.

(C) The Author(s) 2020 